\title{
El valor histórico de las haciendas Malqui-Machay y su incidencia en el aporte de la Identidad Cultural del Cantón La Maná
}

\section{The historical value of the Malqui-Machay farms and its impact on the contribution of the Cultural Identity of the Canton La Maná}

\author{
Peterson Arturo Toapanta Caizaluisa. ${ }^{1}$, Natalia Geoconda Zambrano Cuadro. ${ }^{2}$, Wendy \\ Sabrina Llore Chicaiza. ${ }^{3} \&$ César Enrique Calvopiña León. ${ }^{4}$
}

\begin{abstract}
.
DOI: https://doi.org/10.33262/concienciadigital.v4i1.1521

Introduction. The Malqui-Machay farms located in the parish of Guasaganda, La Maná Canton, have archaeological remains that are known as the last dwelling place of the Inca Atahualpa due to the historical events that have been of importance for Ecuadorian history since it would have been the refuge of the last Incas. These events are a contribution to the cultural identity of the sector thanks to the history it has. However, it has weaknesses in the conservation of archeology and with it the cultural identity of the Lamanense population, this has been a consequence of the devaluation of the infrastructure and by weather conditions. Objective. Preserve the historical value of the Malqui-Machay estates and their impact on the cultural identity of the La Maná Canton. Methodology. For this research, we worked with a sample of 69 people where the opinion of professionals, owners of the haciendas and the population of the Guasaganda parish was taken, this information was collected through field trips using

\footnotetext{
${ }^{1}$ Universidad Técnica de Cotopaxi - Extensión La Maná, carrera de Ingeniería en Ecoturismo. Cotopaxi, Ecuador, peterson.toapanta2035@utc.edu.ec, https://orcid.org/0000-0001-5892-7894

${ }^{2}$ Universidad Técnica de Cotopaxi - Extensión La Maná, carrera de Ingeniería en Ecoturismo. Cotopaxi, Ecuador, natalia.zambrano@utc.edu.ec, https://orcid.org/0000-0002-5678-7725

${ }^{3}$ Universidad Técnica de Cotopaxi - Extensión La Maná, carrera de Ingeniería en Ecoturismo. Cotopaxi, Ecuador,wendy.1lore4499@utc.edu.ec, https://orcid.org/0000-0002-1914-2135

${ }^{4}$ Universidad Técnica de Cotopaxi - Extensión La Maná, carrera de Ingeniería en Ecoturismo. Cotopaxi, Ecuador, cesar.calvopina@utc.edu.ec, https://orcid.org/0000-0003-0551-0950
} 
the interview techniques (5) and survey (64), which were formulated with closed structured questions; The main purpose was to gather information to know the criteria and knowledge of the inhabitants about the historical milestones that happened in the Malqui-Machay estates. Results. According to the study of cultural identity carried out in the Malqui-Machay haciendas, important results are framed, since these sites strengthen the customs and traditions of the population thanks to the events of each hacienda and the legends existing in the surrounding area. With these positive data, we proceed to design an informative cultural heritage guide which contains three sections: informative, legends and conservation. With the fundamental purpose of rescuing part of the cultural identity of the inhabitants of Canton La Maná. Conclusion. Within the Malqui-Machay farms there is a great potential for cultural tourism which contributes to the cultural identity of the inhabitants of the Guasaganda Canton La Maná parish since there is an archeology that was developed thanks to the events and historical memory that have been evident in that place and the legends that have prevailed over time and are an icon for the sector.

Keywords: Malqui-Machay farms, Historical events, Cultural identity, archaeological remains and devaluation.

\section{Resumen.}

Introducción. Las haciendas Malqui-Machay ubicadas en la parroquia de Guasaganda, cantón la Maná, posee vestigios arqueológicos mismos que se los conocen como la última morada del Inca Atahualpa por los sucesos históricos que han sido de importancia para la historia ecuatoriana ya que habría sido el refugio de los últimos Incas. Estos acontecimientos son un aporte para la identidad cultural del sector gracias a la historia que posee, Sin embargo, presenta debilidades en la conservación de la arqueología y con ello la identidad cultural de la población Lamanense, esto ha sido consecuencia de la devaluación de la infraestructura y por las condiciones climáticas. Objetivo. Preservar el valor histórico de las haciendas Malqui-Machay y su incidencia en la identidad cultural del cantón La Maná. Metodología. Para la presente investigación se trabajó con una muestra de 69 personas en donde se tomó la opinión de profesionales, propietarios de las haciendas y la población de la parroquia de Guasaganda, esta información fue levantada a través de salidas de campo mediante las técnica de la entrevista (5) y encuesta (64),mismas que fueron formuladas con interrogantes estructuradas cerradas; El propósito principal fue recabar información para conocer el criterio y el conocimiento de los pobladores acerca de los hitos históricos que sucedieron en las haciendas Malqui-Machay. Resultados. Según el estudio de la identidad cultural realizado en las haciendas Malqui-Machay se enmarcan importantes resultados ya que estos sitios fortalecen las costumbres y tradiciones de la población gracias a los acontecimientos de cada hacienda y las leyendas existentes en 
la zona circundante. Con estos datos positivos se procede a realizar el diseño de una guía informativa de patrimonio cultural la cual contenga tres secciones: informativa, leyendas y conservación. Con el propósito fundamental de rescatar parte de la identidad cultural de los habitantes del cantón La Maná. Conclusión. Dentro de las haciendas Malqui-Machay existe un gran potencial turístico cultural el cual aporta en la identidad cultural de los habitantes de la parroquia Guasaganda Cantón La Maná ya que se encuentra una arqueología que se desarrollaron gracias, a los acontecimientos y a la memoria histórica que se han evidenciado en dicho lugar y a las leyendas que han prevalecido en el tiempo y que son un icono para el sector.

Palabras Claves: Haciendas Malqui-Machay, Sucesos históricos, Identidad cultural, vestigios arqueológicos y devaluación.

\section{Introducción.}

Según la (UNESCO, 2019), en el mundo se han ratificado 193 países que forman parte de la comunidad internacional para la protección del patrimonio mundial cultural y natural del planeta, mismos que representan a 1.121 sitios de los cuales $869(78 \%)$ son culturales, 213 (19 \%) naturales y 39 (3\%) mixtos. La Constitución del Ecuador enfatiza que el país es multiétnico y plurinacional con un patrimonio cultural tangible e intangible (Contitución del Ecuador, 2008), con un amplio potencial turístico cultural denominado diversidad (Abad M, 2013), el mismo que es expresado en innumerables actividades como creencias, ritos, tradiciones, artes, actos festivos y técnica artesanales; Por esta razón el Instituto Nacional de Patrimonio Cultural (INPC, 2014) indica que en los últimos años el país atestigua una efervescencia patrimonial siendo este un factor positivo para la dinamización de la economía de la nación.

En el 2014 la Organización de las Naciones Unidas para la Educación, Ciencia y Cultura (UNESCO) detalla que el sector cultural contribuye al Producto Interno Bruto (PIB) con el $4.76 \%$ y los pobladores que se dedican a las actividades y ocupaciones culturales se encuentran en un porcentaje del $2.2 \%$ representando un alto nivel de producción en el Ecuador siendo este, un ente de gran importancia para potencializar el turismo cultural a nivel nacional. La información expresada con anterioridad ha incrementado el interés por salvaguardar el patrimonio cultural de la provincia de Cotopaxi principalmente en el cantón La Maná en donde se encuentra las haciendas Malqui-Machay en la cual ha sucedido acontecimientos que en su época fueron relevantes para el sector. (Clarembeaux, 2010), menciona que para salvaguardar es indispensable el material didáctico ya que es un hilo conductor para explorar el patrimonio heredado de la memoria, además, la población debe reflexionar sobre los elementos históricos, culturales y sociales del sitio (Palma Peña, 2013). 
En este sitio se enmarcó una investigación arqueológica en el cual participaron varios profesionales nacionales e internacionales como la historiadora ecuatoriana Tamara Estupiñán Viteri y el instituto francés de estudios Andinos quienes participaron en el "Proyecto arqueológico Malqui-Machay", logrando determinar exactamente la ubicación de estas ruinas, en donde se cree que fue la última morada de Atahualpa, púes así lo afirmó la historiadora quien encabezó la primera expedición el 16 de julio de 2004 en Malqui hasta llegar el 26 de junio de 2010 a Machay. (Caiza, 2016).

Después del arduo descubrimiento la historiadora Tamara Estupiñán se ejecuta un estudio a profundidad en donde menciona la relación que tienen las ruinas arqueológicas con los incas quiteños, se lo denomino "Malqui-Machay, la última morada de Atahualpa". En este documento se menciona la hipótesis etnohistórica planteada, Malqui-Machay fue un espacio emblemático ya que los Incas de la resistencia de Quito llevaron los restos mortales de Atahualpa, luego de que fue ejecutado en Cajamarca por los conquistadores españoles. (Estupiñán T. , 2013). Un Artículo publicado por Tamara Estupiñán titulado "MalquiMachay: La cronología del descubrimiento, validación científica, empoderamiento social y puesta en valor" se mencionó la importancia que tienen estos hallazgos para la comunidad cercana es decir Malqui. (Estupiñan Viteri, 2011).

Otra de las investigaciones fue de la arqueóloga Tamara Bray (Wayne University), especialista en los Incas en la frontera norte del Tahuantinsuyo. Quien participa conjuntamente con la historiadora Tamara Estupiñán en corroborar la presencia de los Incas en las ruinas que albergan las haciendas Malqui-Machay. Uno de los artículos relacionados con los incas que fue de importancia para el estudio de la historiadora ecuatoriana fue: "Al final del imperio: el sitio arqueológico Inca-Caranqui en la sierra septentrional del ecuador". En donde menciona el paso de los incas por el territorio ecuatoriano y los acontecimientos del rey del Tahuantinsuyo. (Bray \& Echeverría, 2014). Otros artículos referente a este lugar es: Malqui-Machay: cronología del descubrimiento, validación científica, empoderamiento social y puesta en valor (Estupiñán Viteri, 2011) y Sigchos, el último refugio de los incas, en donde se detalla los asentamientos de estos personajes. (Estupiñán T. , 2011).

En la parroquia de Guasaganda, lugar en donde se encuentran las haciendas Malqui-Machay se han realizado varios estudios, sin embargo, existe un desconocimiento de los pobladores acerca de hitos históricos que han reposado en estas haciendas, pero no asido potenciada. Actualmente estos aspectos han dado como resultado la devaluación de la arqueología y con ello la perdida de la identidad cultural, a pesar de tener el potencial con respecto a historia e infraestructura no han podido enfocarse en un sitio turístico cultural ya que además de la parte tangible la identidad está ligada a la conservación de formas de vida tradicionales. (Vergara Estévez \& Vergara D., 2002). 
Por esta razón el presente estudio se realizó enfocado a fortalecer la identidad cultural de la población lamanense ya que al pasar el tiempo las nuevas generaciones no adoptan la historia que los identifican en una zona Tal como lo expresa (Flores \& Murillo, 2007), la identidad se da gracias a un reencuentro con las tradiciones culturales mismas que contienen este lugar.

\section{Metodología}

Para el presente estudio se tomó en cuenta el criterio de los pobladores de sector circundante es decir 69 personas para identificar el aporte de la arqueología que se encuentra dentro de las haciendas Malqui- Machay, para ello se aplicó la para ello se aplicó la investigación insitu en donde ese obtuvo información verídica de los sucesos históricos que han acontecido en la arqueología que están dentro de las haciendas como lo expresa Rosália la cual menciona que este trabajo interfiere directamente en la calidad de la información a partir de la cual se podrá construir al análisis y llegar a una comprensión más amplia del problema planteado. (Duarte, 2002)

Esta información se recabó en 8 salidas de campo y con la técnica de la entrevista y encuesta se recopilo información de manera general y se constató debilidades de las haciendas para posteriormente identificar estrategias para el rescate de la identidad cultural, mismas que darán lugar a la conservación. Estos datos servirán para el contenido de la guía informativa de patrimonio cultural. También se realizó tres fichas basadas en el formato INPC para el patrimonio cultural inmaterial que son las tradiciones y expresiones orales y dos fichas basadas en el MINTUR para recolectar datos acerca de los atractivos culturales tanto para la hacienda Malqui como la hacienda Machay, esta información se recabo con la técnica de la observación, entrevista y encuesta.

La metodología que se aplicó fue: El método descriptivo, método sintético, método histórico, método de abstracción y método analítico, mismos que sirvieron para recolectar datos de los acontecimientos que sucedieron en estos lugares. Para constatar los acontecimientos que se dieron en aquella época se aplicó el método histórico, mismo que aporta en la reconstrucción del pasado de manera más exacta mediante entrevistas con el tema de los hitos históricos que están dentro de las haciendas con el mismo nombre, la información recabada se obtuvo con las personas que han vivido toda su vida en dicho lugar.

Los datos de las encuestas y entrevistas de carácter exploratorio se recabaron con el método descriptivo, la cual se formuló con preguntas estructuradas cerradas y de selección múltiple aplicadas a una muestra representativa del cantón La Maná. Para interpretar la información recabada se empleó el método sintético en donde se tabuló las preguntas de las encuestas y entrevistas para detectar la necesidad de un diseño para realizar una guía en donde contenga información del patrimonio que poseen las haciendas por su arqueología existente, estas aportaran en la recuperación de la identidad cultural de la parroquia de Guasaganda. Una vez sintetizada la información se analizó la información pertinente y las estrategias para la 
recuperación de la identidad cultural que se incluirán dentro de la guía informativa de las haciendas.

El método de abstracción que se utilizó para la narración de los sucesos que informaron las personas de acuerdo su experiencia, esto se realizó mediante la técnica de la entrevista, la cual aporto en la recolección de datos acerca de los hechos que acontecieron en la infraestructura en aquellos tiempos.

Dentro de la investigación también se aplicó una muestra de cinco entrevistas, tres a los dueños de los terrenos cercanos a la arqueología, una entrevista a un poblador nativo de la parroquia de Guasaganda y otra a la historiadora quien realizo investigaciones acerca de las ruinas de Malqui-Machay, esto se recopilo a través del método de la entrevista. Y para recabar información acerca del valor histórico de las haciendas enfocadas en la identidad cultural se aplicó la técnica de la encuesta mismo que fue dirigida a los beneficiarios directos es decir 64 personas de Guasaganda incluido el recinto Malqui. Por consiguiente, se aplicó la técnica de observación la cual se utilizó para constatar el estado actual de la arqueología ubicadas en las haciendas, de esta manera se identificó varias estrategias para rescatar parte de la identidad cultural de los pobladores de las zonas aledañas.

Estas estrategias para determinar el problema del sitio se aplicó de manera triangular, según (Okuda Benavides \& Gómez Restrepo, 2005), piensa que una de las ventajas de la triangulación es que cuando dos estrategias arrojan resultados muy similares, esto corrobora los hallazgos; pero cuando, por el contrario, estos resultados no lo son, la triangulación ofrece una oportunidad para que se elabore una perspectiva más amplia en cuanto a la interpretación del fenómeno en cuestión, porque señala su complejidad y esto a su vez enriquece el estudio y brinda la oportunidad de que se realicen nuevos planteamientos. Además, los métodos son una herramienta para mejorar el conocimiento sobre la realidad. (Rodríguez Jiménez \& Pérez , 2017)

\section{Resultados.}

Con la metodología aplicada en este proyecto de investigación se pudo obtener un diagnóstico situacional de las haciendas Malqui-Machay, en donde se encuentran la arqueología que contiene la historia de importancia para el recate de la identidad cultural del lugar. Con referencia a las encuestas aplicadas en la parroquia Guasaganda, lugar circundante de la arqueología, en el cual se ejecutó 6 interrogantes que sería útil para determinar la incidencia de la arqueología para la identidad de la población dando como resultado lo siguiente: De acuerdo a la pregunta ¿Conoce la historia que tienen las haciendas MalquiMachay? 
Tabla 1 Conocimiento de la historia

\begin{tabular}{ccc}
\hline Variable & Frecuencia & Porcentaje \\
\hline Conozco demasiado & 6 & $9 \%$ \\
\hline Conozco muy poco & 41 & $64 \%$ \\
\hline No conozco nada & 17 & $27 \%$ \\
\hline Total & $\mathbf{6 4}$ & $\mathbf{1 0 0 \%}$ \\
\hline
\end{tabular}

Nota: Recuperado del proyecto "valor histórico de las haciendas Malqui-Machay y su contribución al fortalecimiento de la identidad cultural" (Llore, 2020)

En la tabla 1 se estableció que la mayoría de la población de la parroquia Guasaganda conoce muy poco acerca de la historia que poseen las haciendas Malqui- Machay, esto se debe a la escasa difusión de la información del lugar, a continuación, está la opción no conozco nada, misma que se debe a la suspensión de la mayoría de las actividades y se van quedando en el olvido, con ello se va perdiendo parte de la identidad cultural de los pobladores. A continuación, se planteó la interrogante ¿Las haciendas Malqui - Machay contribuyen en el rescate de la identidad cultural?

Tabla 2 Contribución de la identidad cultural

\begin{tabular}{ccc}
\hline Variable & Frecuencia & Porcentaje \\
\hline Mucho & 38 & $59 \%$ \\
\hline Poco & 23 & $36 \%$ \\
\hline Nada & 3 & $5 \%$ \\
\hline Total & $\mathbf{6 4}$ & $\mathbf{1 0 0 \%}$
\end{tabular}

Nota: Recuperado del proyecto "valor histórico de las haciendas Malqui-Machay y su contribución al fortalecimiento de la identidad cultural" (Llore, 2020)

De acuerdo a la tabla 2 indica que más del 50\% de los moradores de la parroquia rural Guasaganda están consiente que es de vital importancia las historias que poseen las haciendas Malqui-Machay para la conservación de la identidad cultural ya que esa historia los presenta como habitantes nativos del sector. Seguido del porcentaje de la opción poco, el cual surge por el desconocimiento de dichos lugares y por último no contribuyen nada con un mínimo porcentaje debido a la falta de interés por recuperar parte del patrimonio cultural material e inmaterial. La siguiente interrogante es ¿Existe una devaluación en las ruinas arqueológicas existentes en las haciendas? 
Tabla 3 Existencia de la devaluación

\begin{tabular}{ccc}
\hline Variable & Frecuencia & Porcentaje \\
\hline Mucha devaluación & 34 & $53 \%$ \\
\hline Poca devaluación & 26 & $41 \%$ \\
\hline No existe devaluación & 4 & $6 \%$ \\
\hline Total & $\mathbf{6 4}$ & $\mathbf{1 0 0 \%}$
\end{tabular}

Nota: Recuperado del proyecto "valor histórico de las haciendas Malqui-Machay y su contribución al fortalecimiento de la identidad cultural" (Llore, 2020)

Como se ilustra en la tabla 3 más de la mitad de los pobladores expresan que las ruinas arqueológicas existentes en las haciendas Malqui-Machay tiene una devaluación, misma que es perjudicial ya que con ello se va a perder parte de la identidad cultural de la parroquia Guasaganda, también se perdería un recurso turístico potencial del cantón. Seguido de esta opción se determina que existe poca devaluación ya que los propietarios de las haciendas han realizado actividades para conservar estos lugares. Pero con un mínimo porcentaje de personas piensan que no existe devaluación. A continuación, la siguiente interrogante: ¿Cree usted que se ha perdido el valor histórico de la arqueología existente en las haciendas MalquiMachay?

Tabla 4 Pérdida del valor histórico

\begin{tabular}{ccc}
\hline Variable & Frecuencia & Porcentaje \\
\hline Mucho & 30 & $47 \%$ \\
\hline Poco & 28 & $44 \%$ \\
\hline Nada & 6 & $9 \%$ \\
\hline Total & $\mathbf{6 4}$ & $\mathbf{1 0 0 \%}$
\end{tabular}

Nota: Recuperado del proyecto "valor histórico de las haciendas Malqui-Machay y su contribución al fortalecimiento de la identidad cultural" (Llore, 2020)

En la tabla 4 ilustra que con el transcurso del tiempo se ha perdido el valor histórico de las haciendas por la carencia de documentos en donde se pueda investigar acerca de la historia que posee las ruinas. $\mathrm{Y}$ un grupo de habitantes mencionan que no se ha perdido el valor ya que se puede recuperar, aseveran que con las actividades de recuperación esto se puede rescatar. Con respecto a la pregunta ¿Piensa usted que es importante conservar la identidad Cultural? se detalla la siguiente tabla: 
ISSN: 2600-5859

Tabla 5 Conservación de la Identidad cultural

\begin{tabular}{ccc}
\hline Variable & Frecuencia & Porcentaje \\
\hline Muy importante & 59 & $92 \%$ \\
\hline Importante & 2 & $3 \%$ \\
\hline Poco importante & 3 & $5 \%$ \\
\hline Nada importante & 0 & $0 \%$ \\
\hline Total & $\mathbf{6 4}$ & $\mathbf{1 0 0 \%}$ \\
\hline
\end{tabular}

Nota: Recuperado del proyecto "valor histórico de las haciendas Malqui-Machay y su contribución al fortalecimiento de la identidad cultural" (Llore, 2020)

Los moradores de la parroquia Guasaganda expresan que es muy importante conservar la identidad cultural ya que es lo que les identifican de un lugar, mantener las costumbres y tradiciones es de vital importancia, además de generar actividades turísticas, demostrar a los visitantes lo que tienen. Y con un mínimo porcentaje está la opción poco importante ya que se han aculturado. Para evidenciar la acogida de la población de una guía interpretativa se realizó la siguiente pregunta: ¿Le gustaría contar con una guía de patrimonio cultural en donde se detalle la historia de estos sitios?, con los siguientes datos:

Tabla 6 Contar con una guía de patrimonio

\begin{tabular}{ccc}
\hline Variable & Frecuencia & Porcentaje \\
\hline Mucho & 55 & $86 \%$ \\
\hline Poco & 7 & $11 \%$ \\
\hline Nada & 2 & $3 \%$ \\
\hline Total & $\mathbf{6 4}$ & $\mathbf{1 0 0 \%}$
\end{tabular}

Nota: Recuperado del proyecto "valor histórico de las haciendas Malqui-Machay y su contribución al fortalecimiento de la identidad cultural" (Llore, 2020)

Como se detalla en la tabla 6 la mayoría de personas piensan que es muy importante la creación de un documento en donde se detalle las historias que poseen las haciendas MalquiMachay y las leyendas existentes en la zona circundante. Además, expresan que esto ayudará a captar un mayor número de turistas, mismo que colaborarán en la dinamización de la economía de la parroquia Guasaganda.

Según (Llore, 2020) los resultados de las entrevistas con respectos a la historiadora Tamara Estupiñán dice que las haciendas tienen acontecimientos históricos, los cuales descubrió en el año 2004 tras estudios acerca del personaje Rumiñahui, el hombre más leal de Atahualpa 
se puede corroborar que en el sitio ocurrieron sucesos históricos, importantes para la identidad cultural. Los hallazgos encontrados son los murales rectangulares, estructuradas con piedra cortada y pulida, rasgos que pertenecen al diseño imperial tardío.

Parte de la historia que relató el propietario de la hacienda Machay es la última morada de Atahualpa, misma que se desarrolló hace aproximadamente 10 años de estudios llegando a la conclusión que en el idioma Kichwa Malqui significa momia y Machay morada, es decir la última morada de Atahualpa. (Moncayo, 2019). Actualmente la hacienda pertenecía al cantón Sigchos, por esa razón en la mayoría de escritos, videos, documentales, entre otros. Se menciona que estos hallazgos son de este sitio. Según el Plan de Ordenamiento Territorial La Maná las competencias pasaron al cantón la Maná ubicándose en la parroquia Guasaganda. (PDOT, 2015).

Por otro lado, en la hacienda Malqui existen estudios acerca de los incas en conjunto con la hacienda Machay, sin embrago el propietario se inclina por la historia de los Yumbos con respecto a la arqueología. Y referente a la parte industrial es decir el trapiche con una antigüedad de aproximadamente 104 años siendo una de las primeras, en donde destilaban el licor, esto es parte fundamental para recuperar la identidad cultural. (Rivadeneira, 2019) . Sin embargo, uno de los trabajadores más antiguos de la hacienda Malqui expresa que en la hacienda Malqui existieron los Yumbos e Incas ya que según investigaciones de la arqueóloga Catherine Lara se encontraron tiestos que datan de los tiempos de antes y después de Cristo, esto permitirá obtener más información de los ancestros aportando en el fortalecimiento para la identidad cultural. (Pilaguano, 2019).

Con la información anteriormente mencionada se identificaron cinco estrategias de preservación para arqueología Malqui-Machay la cual incide en la identidad cultural de los habitantes de la parroquia de Guasaganda.

Tabla 7 Estrategia para los rituales ancestrales

Estrategia Objetivo

Fomento de los rituales ancestrales
Rescatar las festividades ancestrales a través de las celebraciones del calendario andino ecuatoriano.

Nota: Recuperado del proyecto "valor histórico de las haciendas Malqui-Machay y su contribución al fortalecimiento de la identidad cultural" (Llore, 2020) 
Como se ilustra en la tabla 1 esta estrategia se desarrolló en base a la afirmación de la historiadora Tamara Estupiñán la cual menciona que la hacienda Machay era un lugar en donde los Incas se reunían para hacer ceremonias, es por ello que en la actualidad el propietario de los terrenos aledaños a la arqueología a formado parte del rescate de algunos rituales ancestrales que se celebraban en aquella época es decir los solsticios y equinoccios, mismos que se celebran en los meses de marzo, junio, septiembre y diciembre. Machay tiene la capacidad de al menos de 150 personas y para el mantenimiento de dicho lugar los turistas aportan con un valor de $5 \$$, a pesar de la reactivación de estas festividades de la sierra ecuatoriana se pretende incluir otras como el Killa Raymi celebrada el 21 de septiembre, está enfocada a la fertilidad de la mujer y la adoración de la luna y el Inti Raymi que es en honor al sol y se celebra cada 22 de junio. Por consiguiente, se menciona la estrategia que involucra a los pobladores.

Tabla 8 Estrategia participativa de la localidad.

Estrategia

Participación activa de la localidad

\section{Objetivo}

Incentivar el interés en los pobladores a través de la inclusión en las actividades de las haciendas Malqui Machay

Nota: Recuperado del proyecto "valor histórico de las haciendas Malqui-Machay y su contribución al fortalecimiento de la identidad cultural" (Llore, 2020)

Esta estrategia sirvió para lograr una participación activa, en la cual los pobladores tendrán reuniones continuas en donde participen todos los pobladores de la comunidad, esto con el propósito que estén informados de las actividades que se van a realizar en las haciendas Malqui-Machay, los dirigentes de la comunidad deben planificar estas reuniones al menos una vez cada semana para recopilar la memoria histórica del adulto mayor y así poder rescatar parte de la identidad cultural transmitiendo estos sucesos de generación en generación.

Las sugerencias para esta estrategia es que se inserten espacios para los pobladores y puedan relatar sus vivencias, esta participación será ejecutada conjuntamente con el gobierno autónomo descentralizado del Cantón la Maná y la academia, mismos que avalen certificados.

Para la conservación del patrimonio cultural es necesario articularse con las entidades públicas y privadas, es por ello que se incluyó esta estrategia. 
Tabla 9 Estrategia de articulación con las entidades.

\section{Estrategia}

Articulación con las entidades públicas y privadas

\section{Objetivo}

Preservar la identidad cultural que yace dentro de los recursos culturales materiales e inmateriales trabajando conjuntamente con las instituciones del cantón.

Nota: Recuperado del proyecto "valor histórico de las haciendas Malqui-Machay y su contribución al fortalecimiento de la identidad cultural" (Llore, 2020)

Como se visualiza en la tabla 3, para ejecutar esta estrategia es necesario conservar la parte arqueología que poseen las haciendas Malqui-Machay, para eso es de suma importancia articular tanto el sector público como GAD La Maná y la Academia con el sector privado es decir operadoras turísticas y agencias de viajes; el trabajo conjunto traerá aspectos positivos para la parte arqueológica y con ello la identidad cultural. Es importante tener este acercamiento con las instituciones ya que colaborarían en la restauración de la arqueología como se detallará a continuación.

Tabla 10 Estrategia de mantenimiento de la arqueología

\begin{tabular}{cl}
\hline Estrategia & \multicolumn{1}{c}{ Objetivo } \\
\hline $\begin{array}{c}\text { Restauración y mantenimiento } \\
\text { de la arqueología }\end{array}$ & $\begin{array}{l}\text { Conservar el patrimonio que posee hitos históricos de } \\
\text { relevancia para la identidad cultural de los pobladores del } \\
\text { lugar. }\end{array}$
\end{tabular}

Nota: Recuperado del proyecto "valor histórico de las haciendas Malqui-Machay y su contribución al fortalecimiento de la identidad cultural" (Llore, 2020)

Las autoridades competentes deben otorgar un presupuesto para mantener los vestigios arqueológicos ya que en la actualidad la estructura está deteriorada por las condiciones climáticas (tropical mega térmico húmedo) y la desvalorización por parte de los pobladores. Por esta razón el mantenimiento debe ser activa, es decir al menos una vez al mes porque el sitio tiende a coparse de maleza, misma razón que afecta a la estructura de las ruinas

En Malqui- Machay perdura el conocimiento acerca de la memoria histórica de lo que aconteció en aquella época, esta sabiduría es importante para preservar la identidad cultural del sitio, por esta razón se detalla la siguiente estrategia: 
Tabla 11 Estrategia para rescatar la memoria histórica

\section{Estrategia Objetivo}

Recopilación de la memoria

Prevalecer el conocimiento de los miembros de la

histórica localidad a través de la recopilación de expresiones orales.

Nota: Recuperado del proyecto "valor histórico de las haciendas Malqui-Machay y su contribución al fortalecimiento de la identidad cultural" (Llore, 2020)

Como se ilustra en la tabla 5, para ejecutar esta estrategia se procederá a recopilar la memoria histórica de los pobladores longevos ya que son los que más experiencia y saberes poseen en cuanto a los hitos históricos que se han desarrollado y las leyendas que poco a poco se han ido olvidando.

\section{Conclusiones.}

- Los vestigios arqueológicos que poseen las haciendas Malqui-Machay tienen potencial Cultural debido a que reposan acontecimientos de los Incas Yumbos y Panzaleos, mismas que han llamado la atención de historiadores, además cuentan con instrumentos de valor cultural como moliendas gigantescas para la destilación del licor artesanal, actividades que hoy en día se han estado olvidando.

- Dentro de la población de la parroquia Guasaganda existe un desconocimiento en cuanto a los sucesos históricos existentes en las haciendas, lo que repercute en la desaparición de parte de su identidad cultural, esto se debe a que la información no se ha transmitido de generación en generación.

- Mediante la investigación se evidenció que las haciendas Malqui-Machay ubicadas en la parroquia rural Guasaganda, aportan en la identidad cultural del sector, debido a los acontecimientos históricos que sucedieron en el lugar, siempre y cuanto esta información sea difundida; por esta razón se realizó una guía de patrimonio cultural de las haciendas Malqui-Machay misma que contribuye en el desarrollo turísticos del cantón.

- Con respecto a la guía informativa de patrimonio cultural de las haciendas MalquiMachay, se evidencia tras la técnica de la encuesta que este documento contribuye en el rescate de la identidad cultural ya que ilustra parte de los sucesos desarrollados en la arqueología ubicados dentro de las haciendas, las cuales son importantes para la población. El documento también refleja la actividad comercial que se realizaba en la parroquia de Guasaganda y que hoy en día se ha ido perdiendo. 


\section{Referencias Bibliográficas.}

Abad M, A. (2013). Las políticas públicas culturales del Ecuador en la época del Sumak Kawsay. SCIELO, 18(26), 57-64. Obtenido de http://www.scielo.org.bo/scielo.php?script=sci_arttext\&pid=S181502762013000100007\&lng=es\&tlng=es.

Bray, T., \& Echeverría, J. (1 de Julio de 2014). Al final del Imperio: El sitio arqueologico Inca-Caranqui en la Sierra Septentrional del Ecuador. Antropología Cuadernos de Investigación(13), 127-170. doi:https://doi.org/10.26807/ant.v0i13.61

Caiza, K. (2016). La Maná Tierra de Encantos (Primera ed.). La Maná, Cotopaxi, Ecuador: IdeaCOM ideas \& comunicación. Recuperado el 2 de Junio de 2019

Clarembeaux, M. (2010). Educación en cine: memoria y patrimonio. Comunicar, XVIII(35), 25-32. Obtenido de https://www.redalyc.org/pdf/158/15815042004.pdf

Contitución del Ecuador. (2008). Contitución del Ecuador. Quito, Ecuador.

Duarte, R. (2002). Pesquisa qualitativa: reflexões sobre o trabalho de campo. Cadernos de Pesquisa. SCIELO(115), 139-154. doi:https://doi.org/10.1590/S010015742002000100005

Estupiñán Viteri, T. (2011). Malqui-Machay: cronología del descubrimiento, validación científica, empoderamiento social y puesta en valor. Open Edition Journals, 593-597. doi:10.4000/bifea.1370

Estupiñan Viteri, T. (1 de Diciembre de 2011). Malqui-Machay: La cronología del descubrimiento, validación científica, empoderamiento social y puesta en valor. Bulletin de l'Institut français d'études andines, 593- 597. Obtenido de https://journals.openedition.org/bifea/1370

Estupiñán, T. (2011). Los Sigchos, el último refugio de los incas quiteños. Una propuesta preliminar. Bulletin de l'Institut français d'études andines, 40(1), 191-204. Obtenido de https://www.redalyc.org/articulo.oa?id=126/12621131007

Estupiñán, T. (2013). Malqui-Machay, La última morada de Atahualpa (1 ed.). Quito.

Flores, \& Murillo. (2007). La identidad cultural del territorio como base de una estrategia de desarrollo sostenible. Revosta Opera(7), 35-54. Obtenido de https://www.redalyc.org/pdf/675/67500703.pdf

INPC. (2014). Revista del Patrimonio Cultural del Ecuador. Revista de INPC, 20. Obtenido de http://mail.inpc.gob.ec/pdfs/Publicaciones/revista5.pdf 
Llore, W. (Febrero de 2020). Valor histórico de las haciendas Malqui-Machay y su contribución al fortalecimiento de la Identidad Cultural. La Maná, Cotopaxi, Ecuador

Moncayo, F. (25 de Junio de 2019). Historia del proyecto Arqueológico realizado en la hacienda Machay. (W. Llore, Entrevistador) La Maná, Cotopaxi, Ecuador.

Okuda Benavides, M., \& Gómez Restrepo, C. (2005). Métodos en investigación cualitativa: triangulación. Colombiana de Psiquiatría, XXXIV(1), 118-124. Obtenido de https://www.redalyc.org/pdf/806/80628403009.pdf

Palma Peña, J. M. (2013). El patrimonio cultural, bibliográfico y documental de la humanidad. Revisiones conceptuales, legislativas e informativas para una educación sobre el patrimonio. SCIELO, 20(58), 31-57. Obtenido de http://www.scielo.org.mx/scielo.php?script=sci_arttext\&pid=S0185$16592013000300003 \& \operatorname{lng}=$ es\&tlng=es.

PDOT. (2015). Plan de Ordenamiento Territorial del Cantón La Maná. La Maná, Cotopaxi, Ecuador.

Pilaguano, S. (3 de Diciembre de 2019). Acontecimeintos de la hacienda Malqui. (W. Llore, Entrevistador) Cotopaxi, Ecuador.

Rivadeneira, F. (4 de Diciembre de 2019). Acontecimeintos de la hacienda Malqui. (W. Llore, Entrevistador) Cotopaxi, Ecuador.

Rodríguez Jiménez, A., \& Pérez , J. A. (2017). Métodos científicos de indagación y de

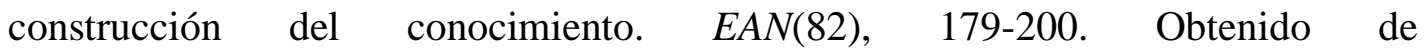
https://dx.doi.org/10.21158/01208160.n82.2017.1647

UNESCO. (2014). Indicadores UNESCO de Cultura para el Desarrollo. Obtenido de Unesco.org:

https://en.unesco.org/creativity/sites/creativity/files/cdis/resumen_analitico_ecuador _0_1.pdf

UNESCO. (2019). Patrimonio Mundial. Obtenido de https://es.unesco.org/themes/patrimonio-mundial

Vergara Estévez, J., \& Vergara D., J. (2002). Cuatro tesis sobre la identidad cultural Latinoamericana. Una reflexión sociológica. Revista de Ciencias Sociales(12), 7792. Obtenido de https://www.redalyc.org/pdf/708/70801206.pdf

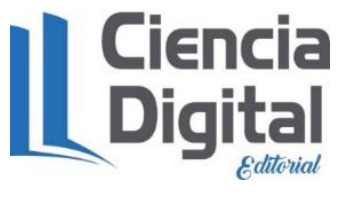




\section{PARA CITAR EL ARTÍCULO INDEXADO.}

Toapanta Caizaluisa, P. A., Zambrano Cuadro, N. G., Llore Chicaiza, W. S., \& Calvopiña León, C. E. (2021). El valor histórico de las haciendas Malqui-Machay y su incidencia en el aporte de la Identidad Cultural del Cantón La Maná. ConcienciaDigital, 4(1), 6-21. https://doi.org/10.33262/concienciadigital.v4i1.1521

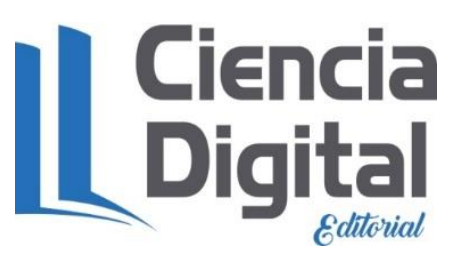

El artículo que se publica es de exclusiva responsabilidad de los autores y no necesariamente reflejan el pensamiento de la Revista Conciencia Digital.

El artículo queda en propiedad de la revista y, por tanto, su publicación parcial y/o total en otro medio tiene que ser autorizado por el director de la Revista Conciencia Digital.
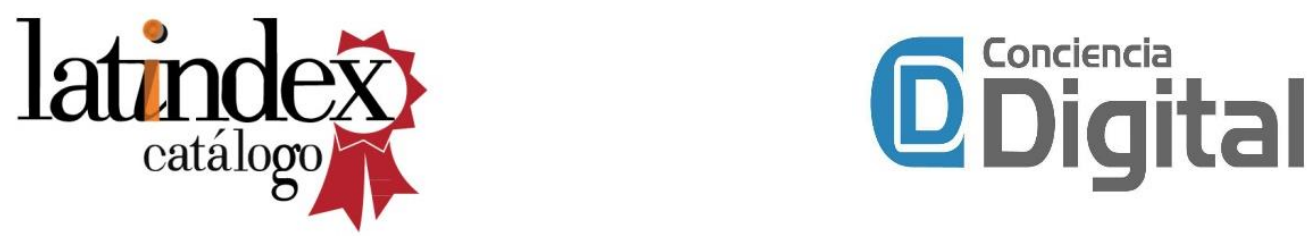